\title{
GCU
}

Glasgow Caledonian

University

University for the Common Good

\section{Disease mechanisms and neuroprotection by tauroursodeoxycholic acid in Rpgr} knockout mice

Zhang, Xun; Shahani, Uma; Reilly, James; Shu, Xinhua

Published in:

Journal of Cellular Physiology

DOI:

$10.1002 / j c p .28519$

Publication date:

2019

Document Version

Author accepted manuscript

Link to publication in ResearchOnline

Citation for published version (Harvard):

Zhang, X, Shahani, U, Reilly, J \& Shu, X 2019, 'Disease mechanisms and neuroprotection by

tauroursodeoxycholic acid in Rpgr knockout mice', Journal of Cellular Physiology, vol. 234, no. 10, pp. 1880118812. https://doi.org/10.1002/jcp.28519

\section{General rights}

Copyright and moral rights for the publications made accessible in the public portal are retained by the authors and/or other copyright owners and it is a condition of accessing publications that users recognise and abide by the legal requirements associated with these rights.

Take down policy

If you believe that this document breaches copyright please view our takedown policy at https://edshare.gcu.ac.uk/id/eprint/5179 for details of how to contact us. 
1 Disease mechanisms and neuroprotection by tauroursodeoxycholic acid in Rpgr knockout mice

2 Xun Zhang ${ }^{1}$, Uma Shahani ${ }^{2}$, James Reilly ${ }^{1}$, Xinhua Shu ${ }^{1,2 *}$

31 Department of Biological and Biomedical Sciences, Glasgow Caledonian University, Cowcaddens

4 Road, Glasgow G4 0BA

52 Department of Vision Science, Glasgow Caledonian University, Cowcaddens Road, Glasgow G4

$6 \quad$ BA

7

8 * Corresponding author Dr Xinhua Shu, Email Xinhua.Shu@gcu.ac.uk

9

10

11

12

13

14

15

16

17

18

19

20

21

22

23

24

25

26

27 


\section{Abstract}

Mutations in the $R P G R$ gene are the predominant cause of retinitis pigmentosa (RP). RPGR plays a critical role as a scaffold protein in the regulation of protein trafficking from the basal body to the axoneme, where the cargoes are transported to the outer segments (OS) of photoreceptors. This trafficking process is controlled directly by intraflagellar transport (IFT) complexes and regulated by the RPGR protein complex, although the precise mechanisms have yet to be defined. We employed an Rpgr conditional knockout (cko) mouse model to investigate the disease mechanisms during retinal degeneration and to evaluate the protective effects of tauroursodeoxycholic acid (TUDCA). Rhodopsin, cone opsins and transducin were mislocalized in Rpgr cko photoreceptors, while localization of NPHP4 to connecting cilia was absent, suggesting that RPGR is required for ciliary protein trafficking. Microglia were activated in advance of retinal degeneration in Rpgr cko mouse retinas. TUDCA treatment suppressed microglial activation and inflammation and prevented photoreceptor degeneration in Rpgr cko mice. Our data demonstrated that TUDCA has therapeutic potential for RPGR-associated RP patients.

KEYWORDS retinitis pigmentosa, RPGR, microglia activation, tauroursodeoxycholic acid, neuroprotection 
Retinitis pigmentosa (RP) is a class of inherited retinal disorder that causes progressive visual impairment and which can lead ultimately to blindness. The worldwide prevalence of RP is about 1 in 4,000, meaning there are more than 1 million people currently living with RP (Hartong et al., 2006). The disorder is characterised by pigmentation in retinal cells that is caused by deposition of materials from retinal pigment epithelium (RPE) cells and degeneration of photoreceptors. In most RP cases, patients lose their peripheral vision and night vision, a process often beginning in adolescence and caused by the death of rod photoreceptors. The subsequent degeneration of cone photoreceptor in later stages results in loss of central vision and colour perception. Some patients, on the other hand, exhibit cone-rod dystrophy, which involves an initial loss of cone photoreceptors followed by degeneration of rods (Hamel, 2007).

Mutations in the retinitis pigmentosa GTPase regulator $(R P G R)$ genes are the major single cause of RP, accounting for up to $20 \%$ of cases in Caucasians (Shu et al., 2007). The $R P G R$ gene has more than 10 alternative transcripts, of which $R P G R^{e x l-19}$ and $R P G R^{O R F 15}$ are well-studied major transcripts (Shu et al., 2005; Vervoort et al., 2000). The $R P G R^{e x l-19}$ transcript has been shown to be widely expressed in all examined tissues in different species, including human, mouse, Xenopus and zebrafish, whereas the $R P G R^{O R F 15}$ transcript is predominantly expressed in the retina and contains a mutation hot spot in the C-terminal exon, called ORF15 (Shu et al., 2006; Raghupathy et al., 2015). The RPGR ${ }^{\text {ex1-19 }}$ isoform is conserved in both vertebrates and invertebrates, whereas, the RPGR ${ }^{\mathrm{ORF} 15}$ isoform is unique to vertebrates (Raghupathy et al., 2015). RPGR forms a complex with other proteins such as RPGR-interacting protein 1 (RPGRIP1) and RPGRIP1-like protein (RPGRIP1L) in the connecting cilium to regulate transport of cargoes such as rhodopsin (Patnaik et al., 2015). Knockout (ko) of Rpgr in mice caused a reduced level of rhodopsin, partially mislocalized blue and green cone opsins, and notable abnormality of newly formed disk membranes at the base of photoreceptors (Hong et al., 2000). Interestingly, significantly increased rhodopsin was mistrafficked in the ventral retina compared to the dorsal retina; however, there was more mistrafficked green opsin in the dorsal retina than in the ventral retina (Charng et al., 2016). Partial mislocalization of rhodopsin and opsin has also been reported in the retinas of XLPRA2 dogs, which carry a two-nucleotide deletion in the RPGR 
exon ORF15 and which exhibited early-onset retinal degeneration (Beltran et al., 2006). Rpgr ko mice were found to exhibit moderate degeneration with approximately $25 \%$ loss of outer nuclear layer (ONL) at 6 months of age (Hong et al., 2000). Rpgr conditional knockout (cko) mice also showed progressive photoreceptor degeneration with $65 \%$ loss of ONL at $\sim 13$ months of age (Huang et al., 2012).

In this study, we investigated photoreceptor death mechanisms and evaluated the neuroprotective effects of tauroursodeoxycholic acid (TUDCA) in Rpgr cko mice. We found that cko mouse retinas had significant increases in cell death and inflammation when compared to wildtype mouse retinas. TUDCA treatment resulted in decreased photoreceptor death and inhibited inflammation in Rpgr cko mouse retinas.

\section{MATERIALS AND METHODS}

\subsection{Animals and TUDCA administration}

Rpgr cko mouse (Huang et al., 2012) were gifted from Professor Wright's lab at MRC Human Genetics Unit, Edinburgh, and bred in the Animal Unit at Glasgow Caledonian University under a 14:10 hour light-dark cycle. Genotyping of Rpgr cko mice was performed by PCR and sequencing. Both wildtype siblings and Rpgr cko mice (male and female) were used for this study. Previous studies have shown that injection of TUDCA at dose $500 \mathrm{mg} / \mathrm{kg}$ is effective in preventing retinal degeneration in RP mouse models (Drack et al., 2012); consequently, this dose was chosen for our study. Six Rpgr cko mice were injected weekly with TUDCA in $0.15 \mathrm{M} \mathrm{NaHCO}_{3}$ at $500 \mathrm{mg} / \mathrm{kg}$ dose intraperitoneally from postnatal days $(\mathrm{P}) 30$ to $\mathrm{P} 120(\mathrm{n}=6)$. Untreated control mice received the same volume of $0.15 \mathrm{M} \mathrm{NaHCO} 3(\mathrm{n}=6)$. The body weight of mice in each group was measured prior to injection each week. There was no significant difference in body weight growth between untreated and TUDCA-treated group (data not shown). The animal experiment was approved by the Animal Ethics and Welfare Committee, Department of Life Sciences, Glasgow Caledonian University in accordance with the UK home office animal care guidelines (Project licence P8C815DC9).

\subsection{Histology and immunohistochemistry}

Enucleated eyes were marked with marker pen to distinguish superior and inferior side and were fixed in $2 \% \mathrm{PFA} / \mathrm{PBS}$ at $4^{\circ} \mathrm{C}$ for $18-24$ hours. The fixed whole eyes then went through $10 \%, 30 \%$, 
$50 \%, 70 \%, 90 \%, 100 \% \times 3$ ethanol each for $1 \mathrm{~h}$, followed by two changes of Histo-Clear (Sigma,

114 USA) and three changes of paraffin in $60^{\circ} \mathrm{C}$ oven. Next, eyes were embedded in paraffin and completely sectioned at $8 \mu \mathrm{m}$ thickness through the vertical meridian. The paraffin sections (within the optic nerve head region) were gradually rehydrated by undergoing two changes of Histo-Clear, two changes of $100 \%$ ethanol ( $5 \mathrm{~min} / \mathrm{each}$ ), $90 \%$ ethanol for $2 \mathrm{~min}, 70 \%$ ethanol for $2 \mathrm{~min}, 50 \%$ ethanol for $2 \mathrm{~min}$ and distilled water for $2 \mathrm{~min}$ before staining with hematoxylin (Sigma, USA) for 8 min. Slides were then washed in running tap water for $20 \mathrm{~min}$ and dehydrated by going through $50 \%$ ethanol for $2 \mathrm{~min}$ and $70 \%$ ethanol for $2 \mathrm{~min}$, then counterstained with eosin (Sigma, USA) for $1 \mathrm{~min}$, followed by further dehydration with $90 \%$ ethanol for $1 \mathrm{~min}$, two changes of $100 \%$ ethanol (5min/each) and two changes of Histo-Clear ( $5 \mathrm{~min} / \mathrm{each})$. Slides were examined and photographed under a light microscope (Olympus, Japan). For measurement of the outer nuclear layer (ONL), two retinal sections of each eye were selected and images were taken from superior and inferior sides of the optic nerve head $(\mathrm{ONH})$; ONL thickness was measured at intervals of $0.2 \mathrm{~mm}$. Five eyes from five individual mice from each group were used for ONL measurement.

Fixed eye samples were processed through a series of sucrose concentrations $(5 \%, 15 \%$ and $20 \%$ ) for 4 hours each step, followed by embedding in OCT (optimal cutting temperature) compound and subsequent freezing. Eyes were completely sectioned at $10 \mu \mathrm{m}$ thickness through the vertical meridian. The sections were rehydrated with wash buffer $(1 \times \mathrm{TBS} / 0.025 \%$ Triton X-100) twice ( $5 \mathrm{~min} / \mathrm{each})$. The sections were then incubated with blocking buffer $(1 \times \mathrm{TBS} / 0.3 \%$ Triton $\mathrm{X}-100 / 5 \%$ sheep serum) for 1 hour at room temperature and incubated with primary antibodies (Table S1) in blocking buffer overnight at $4^{\circ} \mathrm{C}$. Sections were washed 3 times (5min/each) and incubated with FITC-conjugated AF488 or AF594 secondary antibody (1:400 dilution) (Thermo Fisher Scientific, UK) for 1 hour at room temperature. Tissue sections were counterstained with DAPI (Thermo Fisher Scientific, UK) after washing 5 times ( $5 \mathrm{~min} / \mathrm{each}$ ) with wash buffer. Fluorescence images were captured on a ZEISS LSM800 microscope (Zeiss, Germany). To measure the intensity of the fluorescence signal, two retinal sections of each eye were collected: one region $(10 \mu \mathrm{m} \times 10 \mu \mathrm{m}$, under $400 \mathrm{x}$ magnification $)$ in the superior side $(0.4 \mathrm{~mm}$ from the $\mathrm{ONH})$ and one region $(10 \mu \mathrm{m} \times 10 \mu \mathrm{m}$, under $400 \mathrm{x}$ magnification $)$ in the inferior side $(0.4 \mathrm{~mm}$ from 
$141 \mathrm{ONH}$ ) in each section were chosen for fluorescent signal quantification. The intensities of fluorescent

142 signals from the superior region and from the inferior region were averaged for final calculation. Five

143 eyes from each group were used for the quantification.

$144 \quad 2.3$ Quantitative real-time polymerase chain reaction (qRT-PCR)

qRT-PCR was performed with PCR-Platinum ${ }^{\circledR}$ SYBR ${ }^{\circledR}$ Green qPCR SuperMix- UDG kit

(Invitrogen). Briefly, the reactions were set up in a 96-well plate as follows: each $15 \mu 1$ reaction contained $7.5 \mu$ platinum SYBR green qPCR SuperMix-UDG with ROX, $0.4 \mu 110 \mu \mathrm{M}$ of forward and reverse primers, $3 \mu 1$ diluted cDNA sample $(50 \mathrm{ng} / \mu \mathrm{l})$ and $5.7 \mu 1$ of nuclease-free water. The no template controls (NTC), which contained all the components except for the cDNA template, were also set up and topped up to $15 \mu 1$ with nuclease-free water. DNA amplification and fluorescence detection were performed according to previous description (Tohari et al., 2016). Sequences of primers used for qRT-PCR are Caspase 3 forward 5' TGGTGATGAAGGGGTCATTTATG 3'and reverse 5' TTCGGCTTTCCAGTCAGACTC 3'; $I L-\beta$ forward 5' GGAGAACCAAGCAACGACAAAATA 3' and reverse 5' TGGGGAACTCTGCAGACTCAAAC 3'; Gapdh forward 5' GTCTCCTGCGACTTCAGC 3' and reverse 5'

\subsection{Western blotting}

The retinas were lysed and proteins were extracted using T-PER ${ }^{\text {TM }}$ Tissue Protein Extraction Reagent (Thermo Fihser Scientific, UK), and the concentration of protein lysis was measured by protein assay (Biorad, USA). Lysis were incubated with $4 \times$ loading buffer (Thermo Fisher Scientific, UK) at $70^{\circ} \mathrm{C}$ for 10 min before loading. Proteins were separated with precast gel (BioRad, UK) and transferred to the nitrocellulose membrane (GE Healthcare, UK). The membrane was blocked in $5 \%$ milk for $1 \mathrm{~h}$ and incubated with primary antibodies at $4{ }^{\circ} \mathrm{C}$ for about 18 hours. The IRDye secondary antibody (Li-cor, USA) was used for detection of targeting protein.

\subsection{TUNEL assay}

The mouse eyes were fixed and cut as described above. The cryosections $(8 \mu \mathrm{m})$ of mouse eyes were used to perform a TUNEL (Terminal deoxynucleotidyl transferase dUTP nick end labelling) assay to detect apoptotic cells by the DeadEnd ${ }^{\mathrm{TM}}$ Fluorometric TUNEL System ( Promega, UK) 
according to the manufacturer's instruction. Samples were washed with $1 \times$ PBS for 5 min before being fixed by $4 \%$ PFA/PBS and permeabilized with $20 \mu \mathrm{g} / \mathrm{ml}$ Proteinase K solution. Samples were then treated with $\mathrm{rTdT}$ Incubation Buffer for 1 hour at $37^{\circ} \mathrm{C}$ and the reaction was stopped by $2 \times$ SSC. Slides were mounted with DAPI stain and the fluorescence was photographed by ZEISS LSM 800.

\subsection{Statistical analysis}

All data are presented as mean $\pm \mathrm{SD}$. Data were analysed and compared between two groups at different time-points by a non-parametric $t$-test following by Wilcoxon matched-pairs signed rank test with GraphPad Prism software. Data from TUDCA treatment were analysed using one-way ANOVA followed by Bonferroni test. Differences were regarded as statistically significant if $p<0.05$ and are denoted by asterisks $*\left({ }^{*} \mathrm{p}<0.05 ; * * p<0.01 ; * * * p<0.001 ; * * * * \mathrm{p}<0.0001\right)$.

\section{RESULTS}

\subsection{Abnormal ciliary trafficking in Rpgr cko mouse retinas}

Using Sanger sequencing we found that a total of $3189 \mathrm{bp}$ was deleted in the Rpgr cko mice, including proximal promoter, exons 1-3, and resulted in absence of RPGR expression and localization to connecting cilia of cko mouse photoreceptors (data not shown). To investigate the ciliary trafficking of phototransduction components in the Rpgr cko retina, immunohistochemistry of eye cryosections from one month-old mice was performed with anti-opsins, anti-transducin and antiGRK1 antibodies. Rhodopsin was localised to outer segments of WT photoreceptors; in Rpgr cko mouse photoreceptor the rhodopsin was partially mislocalized to the connecting cilium. Similarly, red/green opsin and blue opsin were mistrafficked and accumulated in the connecting cilium, the perinuclear space and the outer plexiform layer (Figure 1). However, there was no difference in GRK1 localization in WT and Rpgr cko photoreceptors (data not shown).

NPHP4 is a component of RPGR protein complex by direct interaction with RPGRIP1 (Patnaik et al., 2015; Roepman et al., 2005). Mutations in NPHP4 caused nephronophthisis and Senior-Løken syndrome (a combination of nephronophthisis and RP) (Mollet et al., 2012; Otto et al., 2002). NPHP4 is localized to the connecting cilium of photoreceptors, a localization that is dependent on RPGRIP1 (Patil et al., 2012; Roepman et al., 2005; Won et al., 2011). We performed immunohistochemistry in 
paraffin sections of retina to detect NPHP4 localization. In WT retina, NPHP4 was present in the connecting cilium along with acetylated $\alpha$ tubulin. However, in Rpgr cko retina NPHP4 was absent from the connecting cilium (Figure 2A). We also examined NPHP4 expression in retinas by Western blotting and found that NPHP4 expression was significantly decreased at aged 3 months and above (Figure 2B,C; Figure S1).

\subsection{Photoreceptor cell degeneration in Rpgr cko mice}

To assess photoreceptor cell degeneration throughout ageing, we performed hematoxylin \& eosin staining with paraffin sections of WT and Rpgr cko mouse eyes at ages 1, 3, 6, and 12 months and measured the thickness of the outer nuclear layer (ONL) at five different points on both superior and inferior sides of the retina. ONL thickness started to show a significant decrease in 3-month old Rpgr cko mice and was further markedly decreased at 6 months old, compared to age-matched WT mice. Only 2-3 layers of photoreceptor nuclei remained in 12-month old Rpgr cko mice (Figure S2). We also measured the length of photoreceptor outer segments in WT and Rpgr cko mice at different age points. At one-month old, the length of OSs was similar between WT and Rpgr cko mice; from the age of 3 months, however, OSs in cko mice were significantly shorter than those of WT mice (Figure S3).

Decreased thickness of the ONL in Rpgr cko mice was surmised to be due to death of photoreceptor cells. We performed a TUNEL assay to detect the total photoreceptor death at different age points. Significantly increased photoreceptor cell death was present in cko mouse retinas from 3 months of age and peaked at 6 months. In the age-matched WT retina, no significant cell death was observed (Figure 3). To determine whether photoreceptor cell death is caspase-dependent, we employed immunohistochemistry to detect activation of Caspase-3, the key component of the caspasedependent cell death pathway, and found that cleaved caspase- 3 was present in the outer plexiform layer (OPL) and ONL at age 3, 6 and 12 months (Figure S4).

\subsection{Microglia were activated at the early stage of retinal degeneration}

Microglia are immune cells resident in the central nervous system, including the retina (Li et al., 2015). In the healthy mammalian retina, microglia are in resting form, releasing anti-inflammatory factors to maintain homeostasis of the retina; however, they can be activated to the ramified form to 
mediate phagocytosis in disease conditions (Karlstetter et al., 2015). To investigate if there was microglial activation in Rpgr cko mouse retinas, Iba-1, a biomarker of microglia, was measured by immunohistochemistry in cryosections from WT and Rpgr cko mouse eyes. In the latter the microglia were activated and migrated into the ONL as early as 1 month old, whereas in the age-matched WT retina they were present in the OPL (Figure 4).

To explore the consequence of microglial activation in Rpgr cko mouse retina, we examined the microglia-mediated inflammation. In macrophages or dendritic cells, the inflammasomes are assembled by scaffold protein NLRP3 with ASC adaptor and caspase-1 via PYD and CARD domain (Schroder \& Tschopp, 2010). In the Rpgr cko retina, we found NLRP3 colocalized with Iba-1 in the ONL (Figure 5), indicating the formation of inflammasomes in activated microglia. The result suggests that microglia-mediated inflammation might contribute to the retinal degeneration in Rpgr cko mice.

\subsection{TUDCA treatment ameliorated retinal degeneration in Rpgr cko mice}

Tauroursodeoxycholic acid (TUDCA), the bile acid, has been widely used as an anti-apoptotic, antiinflammatory and antioxidant compound (Pardue \& Allen, 2018). TUDCA has been demonstrated to cause inhibition of retinal microglia activation and preservation of retinal structure and visual function (Pardue \& Allen, 2018). To test the protective efficacy of TUDCA in Rpgr cko retina, Rpgr cko mice were intraperitoneally injected weekly with TUDCA $\left(500 \mathrm{mg} / \mathrm{kg}\right.$ in $\left.0.15 \mathrm{M} \mathrm{NaHCO}_{3}\right)$ from P30 to P120 (n=6). Untreated control Rpgr cko mice received an equivalent volume of $0.15 \mathrm{M} \mathrm{NaHCO}_{3}$ $(\mathrm{n}=6)$. The thickness of the ONL was significantly increased in TUDCA-treated Rpgr cko mice when compared to untreated Rpgr cko mice (Figure 6A). The number of photoreceptors was significantly decreased in cko mice when compared to the wildtype mice; TUDCA treatment resulted in a higher number of photoreceptors compared to untreated cko mice (Figure S5). The decreased number of photoreceptor cells is possibly due to cell death; to investigate this we carried out a TUNEL assay and found that the number of photoreceptors undergoing cell death was notably reduced in the treated retina (Figure 6B). The caspase-dependent apoptosis pathway in cko mouse retina was possibly inhibited by TUDCA treatment, since expression of caspase 3 on both protein and mRNA levels was notably decreased (Figure 6C, Figure S6A). In addition, TUDCA treatment significantly ameliorated 
microglia activation by reducing the infiltration of activated microglia into the ONL (Figure 7A). The inflammasome formation in activated microglia was also supressed (Figure 7B), followed by decreased expression of matured inflammatory cytokine IL-1 $\beta$ (Figure 7C, Figure S6B).

\section{DISCUSSION}

In the current study we investigated mistrafficking of photoreceptor proteins, photoreceptor cell death and microglial activation, and evaluated the protective effects of TUDCA in the retina of Rpgr cko mice. We found that rhodopsin was partially mislocalized to the connecting cilia and that cone opsins were also partially mistrafficked to the cone inner segment, cell body and synapse (Figure 1). Partial mislocalization of rhodopsin and cone opsins in photoreceptors has also been reported in Rpgr ko mice (Hong et al., 2000; Charng et al., 2016). $\alpha$-transducin, which operates in rod visual function, was also partially mislocalized in Rpgr cko mice. In fact, mislocalization of rhodopsin, cone opsins and $\alpha$ transducin has also been reported in Rrgrip1 ko mice and mutant zebrafish (Raghupathy et al., 2017; Won et al., 2009). NPHP4 is mutated in nephronophthisis with vision defects (Mollet et al., 2002) and directly interacts with RPGRIP1. Loss of NPHP4 or RPGRIP1 in mice resulted in abnormal development of outer segments (Won et al., 2009; 2011). Ptil et al. (2012) reported loss of NPHP4 localization to connecting cilia in RPGRIP1 ko mice. We also found that NPHP4 lost localization to connecting cilia in Rpgr cko mice and was decreased in cko retinas from 3 months old (Figure 2), possibly due to loss of photoreceptors, since photoreceptor death in cko mice was noticed from 3 months old and above. A proteomic study by Rao et al (2015) reported that proteins involved in ubiquitin-proteasome system or cilia function were decreased in Rpgr ko photoreceptor cilia and that some of these proteins were partially mislocalized (Rao et al., 2015). Our recent study also showed that RPGR protein complex regulated proteasome activities. These data suggest that the RPGR protein complex functions in ciliary protein trafficking and in maintenance of photoreceptor structure and function.

Microglia have been reported to mediate retinal degeneration (Karlstetter et al., 2015). Activated microglia have been detected in the retinas of RP patients (Gupta et al., 2003). Microglial activation has also been observed in preclinical RP rodent models including Pde6-alpha (Pde6a) and Pde6-beta (Pde6b) mutant mice, Cngb1 knockout mice, homozygous P23H rats and Royal College of Surgeons 
rats (Blank et al., 2018; Peng et al., 2014; Roche et al., 2016; Roque et al., 1996; Yoshida et al., 2013; Zeiss and Johnson, 2004; Zeng et al., 2005; Zhang et al., 2018; Zhao et al., 2015). In fact, microglia activation occurs prior to the initiation of photoreceptor degeneration. In rd10 mice, retinal microglial activation was initiated at postnatal day (P) 16 while the photoreceptor apoptosis started at P19 (Peng et al., 2014); a recent report showed that microglia were activated as early as P5 in rd10 mouse retinas (Roche et al., 2016). Early microglial activation ahead of photoreceptor cell death has also been reported in rd1 and Cngb1 knockout mice (Blank et al., 2018; Zeiss et al., 2004). We observed early microglial activation in Rpgr cko mouse retinas at the age of one month when photoreceptor death had yet to be initiated (Figures 3 and 4). The data demonstrate that early microglial activation in retinas is a general feature of inherited retinal degeneration, possibly independent of genetic causes. The initiation of microglial activation in inherited retinal degeneration is not clear; it is possibly induced by toxic factors from pre-apoptotic photoreceptors. When photoreceptor death was halted by a genetic rescue, the activated microglia disappeared in photoreceptor degeneration sites and possibly became ramified (Zhang et al., 2018), indicating that photoreceptor death regulates microglial activation. It is presumed that activated microglia phagocytose pre-apoptotic mutant photoreceptors and secrete proinflammatory cytokines such as TNF- $\alpha$ and IL-1 $1 \beta$, which accelerate retinal degeneration (Karlstetter et al., 2015). Zhao et al. (2015) reported that both genetic depletion of microglia and suppression of microglial phagocytosis slowed rod cell death in rd10 mice. They also observed that photoreceptor cell death was suppressed following inhibition of IL- $1 \beta$ signalling using an IL-1 receptor antagonist; IL-1 $\beta$ was also significantly lower in mouse retina with genetic depletion of microglia when compared to control mice (Zhao et al., 2015). An early study reported that TNF- $\alpha$ expression was predominantly upregulated in activated microglia in rd mice (Zeng et al., 2005). We also found inflammasome formation and predominant expression of IL-1 $\beta$ in activated microglia in Rpgr cko mouse retina (Figures 6 and 7). These data suggest that activated microglia-mediatedinflammation is involved in photoreceptor degeneration.

TUDCA has shown protective effects in a wide range of diseases including liver disease, kidney stones and gallstones, cardiovascular disease, diabetes, and neurodegenerative diseases (Pardue and Allen, 2018). TUDCA has also been used to treat rodent models of inherited retinal degeneration 
including rd1, rd10, rd16, Bardet-Biedl syndrome 1 and $\mathrm{Lrat}^{-/}$mice, and transgenic Rhodopsin $\mathrm{P} 23 \mathrm{H}$ rats; treated animals showed well-preserved retinal structure and improved visual function (Boatright et al., 2006; Drack et al., 2012; Fernandez-Sanchez et al., 2011; Noailles et al., 2014; Phillips et al., 2008; Zhang et al., 2012). It is proposed that protection of photoreceptor death by TUDCA treatment is due to improved protein folding and trafficking, reduced oxidative and ER stress, suppression of inflammation, decreased apoptosis, and increased RPE phagocytosis (Pardue and Allen, 2018). A recent report demonstrated that TUDCA inhibited microglial activation and decreased microglial distribution in outer retinal layers in Rhodopsin P23H homozygous rats (Noailles et al., 2014). We found that TUDCA treatment preserved the retinal structure of Rpgr cko mice (Figure 6), possibly through inhibition of apoptotic cell death since the number of apoptotic cells was significantly decreased in TUDCA-treated Rpgr cko mice when compared to untreated controls and since expression of the key component of apoptotic pathway, Caspase 3, was markedly decreased in TUDCA-treated Rpgr cko mouse retina (Figure 7). Zhang et al. (2012) also showed that TUDCA treated $\mathrm{Lrat}^{-/}$mice had decreased apoptosis with disappearance of activated Caspase 3. We also found that TUDCA treatment inhibited microglial activation and suppressed microglial migration to outer retinal layers (Figure 7). TUDCA treatment also reduced the NLRP3 inflammasome formation in activated microglia and decreased IL-1 $\beta$ expression in treated Rpgr cko mouse retinas (Figure 7), which is consistent with previous studies that showed that TUDCA inhibited expression of inflammatory factors and promoted anti-inflammatory transcripts in rat microglia (Yanguas-Casás et al., 2017).

In conclusion, we observed defective ciliary protein trafficking and early microglial activation in the retinas of Rrpgr cko mice. TUDCA treatment inhibited microglial activation and inflammation, resulting in preservation of retinal structure in Rpgr cko mice.

\section{ACKNOWLEDGEMENTS}

XZ was funded by a Fight for Sight PhD studentship (1419/20). We would like to thank the Rosetrees Trust (M160, M160-F1, M160-F2) and National Eye Research Centre (SCIAD063, SAC037) for supporting this work. 
338 The authors declare there are no conflicts of interest.

\section{AUTHOR CONTRIBUTIONS}

$\mathrm{XZ}$ performed the experiments. XS and US supervised the project. XZ, JR and XS analysed the data and wrote the manuscript.

\section{REFERENCES}

Appelbaum, T., Becker, D., Santana, E., \& Aguirre, G. D. (2016). Molecular studies of phenotype variation in canine RPGR-XLPRA1. Molecular Vision, 22, 319-331.

Beltran, W.A., Hammond, P., Acland, G. M., \& Aguirre, G. D. (2006) A frameshift mutation in RPGR exon ORF15 causes photoreceptor degeneration and inner retina remodeling in a model of Xlinked retinitis pigmentosa. Investigative Ophthalmology \& Visual Science, 47, 1669-1681. Blank, T., Goldmann, T., Koch, M., Amann, L., Schön, C., Bonin, M., ... Michalakis, S. (2018). Early microglia activation precedes photoreceptor degeneration in a mouse model of CNGB1-linked retinitis pigmentosa. Frontiers in Immunology, 8, 1930.

Boatright, J. H., Moring, A. G., McElroy, C., Phillips, M. J., Do, V.T., Chang, B., ... Pardue, M. T. (2006). Tool from ancient pharmacopoeia prevents vision loss. Molecular Vision, 12, 1706-1714. Charng, J., Cideciyan, A. V., Jacobson, S. G., Sumaroka, A., Schwartz, S. B., Swider, M., ... Swaroop, A. (2016) Variegated yet non-random rod and cone photoreceptor disease patterns in RPGR-ORF15-associated retinal degeneration. Human Molecular Genetics, 25, 5444-5459. Drack, A.V., Dumitrescu, A.V., Bhattarai, S., Gratie, D., Stone, E. M., Mullins, R., \& Sheffield, V.C. (2012). TUDCA slows retinal degeneration in two different mouse models of retinitis pigmentosa and prevents obesity in Bardet-Biedl syndrome type 1 mice. Investigative Ophthalmology \& Visual Science, 53, 100-106. Fernandez-Sanchez, L., Lax, P., Pinilla, I., Martin-Nieto, J., \& Cuenca, N. (2011). Ophthalmology \& Visual Science, 52, 4998-5008. 
Gupta, N., Brown, K. E., \& Milam, A. H. (2003). Activated microglia in human retinitis pigmentosa,

365 late-onset retinal degeneration, and age-related macular degeneration. Experimental Eye Research, 76, 463-471.

Hamel, C. P. (2007) Cone rod dystrophies. Orphanet Journal of Rare Disease, $2,7$.

Hartong, D. T., Berson, E. L., \& Dryja, T. P. (2006) Retinitis pigmentosa. Lancet, 368, 1795-809.

Hong, D. H., Pawlyk, B. S., Shang, J., Sandberg, M. A., Berson,E.L., \& Li, T. (2000) A retinitis pigmentosa GTPase regulator (RPGR)-deficient mouse model for X-linked retinitis pigmentosa (RP3). Proceedings of the National Academy of Sciences of the United States of America, 97, 36493654.

Huang, W. C., Wright, A. F., Roman, A. J., Cideciyan, A. V., Manson, F. D., Gewaily, D. Y., ... Jacobson, S. G. (2012) RPGR-associated retinal degeneration in human X-linked RP and a murine model. Investigative Ophthalmology \& Visual Science, 53, 5594-5608.

Karlstetter, M., Scholz, R., Rutar, M., Wong, W. T., Provis, J. M., \& Langmann, T. (2015) Retinal microglia: just bystander or target for therapy? Progress in Retinal and Eye Research, 45, 30-57.

Li, L., Eter, N., \& Heiduschka, P. (2015) The microglia in healthy and diseased retina. Experimental Eye Research, 136,116-130.

Mollet, G., Salomon, R., Gribouval, O., Silbermann, F., Bacq, D., Landthaler, G., ... Saunier, S. (2002) The gene mutated in juvenile nephronophthisis type 4 encodes a novel protein that interacts with nephrocystin. Nature Genetics, 32, 300-305.

Noailles, A., Fernandez-Sanchez, L., Lax, P., \& Cuenca, N. (2014). Microglia activation in a model of retinal degeneration and TUDCA neuroprotective effects. Journal of Neuroinflammation, 11, 186.

Otto, E., Hoefele, J., Ruf, R., Mueller, A. M., Hiller, K.S., Wolf, M. T., ... Hildebrandt, F. (2002) A gene mutated in nephronophthisis and retinitis pigmentosa encodes a novel protein, nephroretinin, conserved in evolution. American Journal of Human Genetics, 71, 1161-1167.

Pardue, M. T., \& Allen, R. S. (2018) Neuroprotective strategies for retinal diseases. Progress in Retinal and Eye Research, 65, 50-76.

Patil, H., Tserentsoodol, N., Saha, A, Hao, Y., Webb, M., \& Ferreira, P. A. (2012) Selective loss of RPGRIP1-dependent ciliary targeting of NPHP4, RPGR and SDCCAG8 underlies the degeneration of photoreceptor neurons. Cell Death \& Disase, 3, e355. 
393

394

395

396

397

398

399

400

401

402

403

404

405

406

407

408

409

410

411

412

413

414

415

416

417

418

419

Patnaik, S. R., Raghupathy, R. K., Zhang, X., Mansfield, D., \& Shu, X. (2015) The Role of RPGR and Its Interacting Proteins in Ciliopathies. Journal of Ophthalmology, 2015, 414781.

Patnaik, S. R., Zhang, X., Biswas, L., Akhtar, S., Zhou, X., Kusuluri, D. K., ... Shu, X. (2018) RPGR protein complex regulates proteasome activity and mediates store-operated calcium entry. Oncotarget, 9, 23183-23197.

Peng, B., Xiao, J., Wang, K., So, K. F., Tipoe, G. L., \& Lin, B. (2014) Suppression of microglial activation is neuroprotective in a mouse model of human retinitis pigmentosa. Journal of Neuroscience, 34, 8139-8150.

Phillips, M. J., Walker, T. A., Choi, H. Y., Faulkner, A. E., Kim, M. K., Sidney, S. S., ... Pardue, M.T. (2008). Tauroursodeoxycholic acid preservation of photoreceptor structure and function in the rd10 mouse through postnatal day 30 . Investigative Ophthalmology \& Visual Science, 49, 2148 2155.

Raghupathy, R. K., Gautier, P., Soares, D. C., Wright, A. F., \& Shu, X. (2015) Evolutionary Characterization of the Retinitis Pigmentosa GTPase Regulator Gene. Investigative Ophthalmology \& Visual Science, 56, 6255-6264.

Raghupathy, R. K., Zhang, X., Liu, F., Alhasani, R.H., Biswas, L., Akhtar, S., ... Shu, X. (2017) Rpgrip1 is required for rod outer segment development and ciliary protein trafficking in zebrafish. Scientific Reports, 7, 16881.

Rao, K. N., Li, L., Anand, M., \& Khanna, H. (2015) Ablation of retinal ciliopathy protein RPGR results in altered photoreceptor ciliary composition. Scientific Reports, 5, 11137.

Roche, S. L., Wyse-Jackson, A. C., Byrne, A. M., Ruiz-Lopez, A. M., \& Cotter, T. G. (2016)

Alterations to retinal architecture prior to photoreceptor loss in a mouse model of retinitis pigmentosa. The International Journal of Developmental Biology, 60, 127-139.

Roepman, R., Letteboer, S. J., Arts, H. H., van Beersum, S. E., Lu, X., Krieger, E., ... Cremers, F. P. (2005) Interaction of nephrocystin-4 and RPGRIP1 is disrupted by nephronophthisis or Leber congenital amaurosis-associated mutations. Proceedings of the National Academy of Sciences of the United States of America, 102, 18520-18525. 

photoreceptors degenerate in Royal College of Surgeons rats. Investigative Ophthalmology \& Visual Science, 37, 196-203.

Schroder, K., \& Tschopp, J. (2010) The Inflammasomes. Cell, 140, 821-832. RPGR mutation analysis and disease: an update. Human Mutation, 28, 322-328. RPGR ORF15 isoform co-localizes with RPGRIP1 at centrioles and basal bodies and interacts with nucleophosmin. Human Molecular Genetics, 14, 1183-1197.

Shu, X., Zeng, Z., Eckmiller, M. S., Gautier, P., Vlachantoni, D., Manson, F. D., ... Wright, A. F. (2006) Developmental and tissue expression of Xenopus laevis RPGR. Investigative Ophthalmology \& Visual Science, 47, 348-356. Zebrafish Rpgr is required for normal retinal development and plays a role in dynein-based retrograde transport processes. Human Molecular Genetics, 19, 657-670. (2012) Rd9 is a naturally occurring mouse model of a common form of retinitis pigmentosa caused by mutations in RPGR-ORF15. PLoS One, 7, e35865.

Tohari, A., Zhou, X., \& Shu, X. (2016) Protection against oxidative stress by vitamin D in cone cells. Cell Biochemistry and Function, 34, 82-94.

440 Vervoort,R.., Lennon, A., Bird, A. .C., Tulloch,B., Axton, R., Miano, M. G., ... Wright, A. F. (2000)

441 Mutational hot spot within a new RPGR exon in X-linked retinitis pigmentosa. Nature Genetics, 25, $462-466$. 
447 Nishina, P. M. (2011) NPHP4 is necessary for normal photoreceptor ribbon synapse maintenance and outer segment formation, and for sperm development. Human Molecular Genetics, 20, 482-496.

452 Yoshida N, Ikeda Y, Notomi S, Ishikawa K, Murakami Y, Hisatomi T, Enaida H, Ishibashi T.

453 Laboratory evidence of sustained chronic inflammatory reaction in retinitis pigmentosa.

454 Ophthalmology. 2013;120(1):e5-12.

Zeiss, C. J., \& Johnson, E. A. (2004). Proliferation of microglia, but not photoreceptors, in the outer nuclear layer of the rd-1 mouse. Invest Ophthalmol Vis Sci. 2004;45(3):971-6.

Zeng HY, Zhu XA, Zhang C, Yang LP, Wu LM, Tso MO. Identification of sequential events and factors associated with microglial activation, migration, and cytotoxicity in retinal degeneration in rd mice. Invest Ophthalmol Vis Sci. 2005 Aug;46(8):2992-9. Pigmentosa. Mol Ther. 2018. pii: S1525-0016(18)30273-9. a mouse model of Leber congenital amaurosis. Investig. Ophthalmol. Vis. Sci., 53, 3349-3356. Zhao L, Zabel MK, Wang X, Ma W, Shah P, Fariss RN, Qian H, Parkhurst CN, Gan WB, Wong WT. Microglial phagocytosis of living photoreceptors contributes to inherited retinal degeneration. EMBO 
475 Figure 1 Loss of RPGR caused mislocalization of opsins and transducin. Cryosections of retinas at 1month (M) old were stained for rhodopsin (green), red/green opsin (red), blue opsin (red), transducin (red), and nuclei were counterstained with DAPI (blue). Opsins and transducin were localized to outer segments (OSs) in wildtype (WT) mouse retinas. Rhodopsin was mislocalized to inner segment (IS) in Rpgr cko retina, while red/green opsin, blue opsin and transducin exhibited mislocalization to both the IS and outer nuclear layer (ONL) in Rpgr cko retina. Arrowheads indicate mislocalized rhodopsin, red/green and blue opsins in the respective images.

Figure 2 NPHP4 was absent from connecting cilia in Rpgr cko mice. (A) Paraffin sections of mouse retina were stained for NPHP4 (green) and connecting cilium (CC) marker acetylated $\alpha$ tubulin (red). NPHP4 was localized to connecting cilia and partially co-localized with acetylated $\alpha$ tubulin in wildtype (WT) retina but was absent from Rpgr cko (RPGR KO) mouse retinas (n=3). (B) Western blotting with total retina lysis of three WT and Rpgr cko mice was performed. (C) NPHP4 (158 kDa) protein level was significantly decreased in Rpgr cko mouse retinas at 3, 6 and 12 months (M) old. Data are from two separate Western blots. Statistical comparisons were performed by a nonparametric $t$-test followed by Wilcoxon matched-pairs signed rank test. ${ }^{*} \mathrm{p}<0.05$; ns, no significance. Figure 3 Cell death occurred in Rpgr cko retinas. (A) TUNEL assay was carried out with cryosections of WT and Rpgr cko retinas at 1M, 3M, 6M and 12M old. Green signal represented the DNA fragmentation in photoreceptors undergoing cell death. Arrowheads indicate nuclei of apoptotic cells. (B) Quantification of percentage of apoptotic cells in total photoreceptors. Blue line represents wildtype (WT) group and red line represents Rpgr cko group. Cell death signal significantly increased at $3 \mathrm{M}$ old in cko retinas and peaked at $6 \mathrm{~m}$ old. Data were collected and analysed by a non-parametric $t$-test followed by Wilcoxon matched-pairs signed rank test $(\mathrm{n}=5) .{ }^{*} \mathrm{p}<0.05,{ }^{*} \mathrm{p}<0.01,{ }^{* * *} \mathrm{p}<0.001$; ns, no significance.

Figure 4 Microglia were activated and induced inflammation at an early stage. (A) Immunostaining of microglia marker Iba-1 (red) was performed with cryosections at different ages. In Rpgr cko retina, microglia were stimulated into a ramified shape and infiltrated the outer nuclear layer (ONL) as early 
502 photoreceptors in both WT and Rpgr cko retina was quantified with ZEN and analysed with a non503 parametric $t$-test followed by Wilcoxon matched-pairs signed rank test $(\mathrm{n}=5) .{ }^{*} \mathrm{p}<0.05$.

504 Figure 5 Activation of inflammation pathway in photoreceptor cell death. Co-immunostaining was performed for detection of Iba-1 (red) and NLRP3 (green). The overlap of Iba-1 and NLRP3 was observed in the outer nuclear layer (ONL) in cko retina at different ages; in wildtype (WT) retinas, they were found only in outer plexiform layer (OPL). Arrowheads indicate Iba-1/NLRP3. Figure 6 TUDCA protected the morphological loss of photoreceptors. TUDCA treatment ameliorated retinal degeneration in Rpgr cko mice. A total of six eyes (one eye from each treated or untreated mouse) were used for cryosectioning and immunostaining. Nuclei in retina were counterstained with DAPI (blue). (A) Outer nuclear layer (ONL) of untreated Rpgr cko retinas ( $\mathrm{n}=6$ ) was significantly thinner than ONL of TUDCA-treated retinas. (B) TUDCA attenuated cell death of photoreceptors as determined by TUNEL assay, indicating that less cell death occurred in ONL after TUDCA treatment. Arrowheads indicate nuclei of apoptotic cells. (C) TUDCA treatment reduced cleaved-caspase 3 protein (red) expression detected by immunostaining. Arrowheads indicate activated caspase 3 . Statistical comparisons were performed using one-way ANOVA followed by Bonferroni test. ${ }^{*} \mathrm{p}<0.05$, $* * \mathrm{p}<0.01, * * * \mathrm{p}<0.001$.

Figure 7 TUDCA treatment reduced the activity of microglia in the photoreceptor layer and ameliorated inflammation. A total of six eyes (one eye from each treated or untreated mouse) were used for cryosectioning and immunostaining. (A) Iba-1 (red) represents the microglia state. In the TUDCA treated group, invasion of microglia (arrowheads) in ONL was significantly reduced compared to the untreated group. (B) Co-immunostaining of Iba-1 (red) and NLRP3 (green) was performed. In the TUDCA treated group, NLRP3 expression was restricted to the outer plexiform layer whereas in the untreated group was present also in the ONL. Red arrowheads indicate microglia; green arrowheads indicate NLRP3. Broken lines are used to separate different retinal layers. (C) Immunostaining of IL-1 $\beta$ (green, arrowheads) was performed and showed that TUDCA treatment dramatically reduced IL-1 $\beta$ in retinas. Total RNAs were extracted from mouse retinas (one retina 
528 from each eye of individual treated or untreated mice; in total 6 retinas) were used for cDNA

529 synthesis and for qRT-PCR. Statistical comparisons were performed using one-way ANOVA

530 followed by Bonferroni test. $* \mathrm{p}<0.05,{ }^{* * *} \mathrm{p}<0.001$.

531

532

533

534

535

536

537

538

539

540

541

542

543

544

545

546

547 
Figure 1

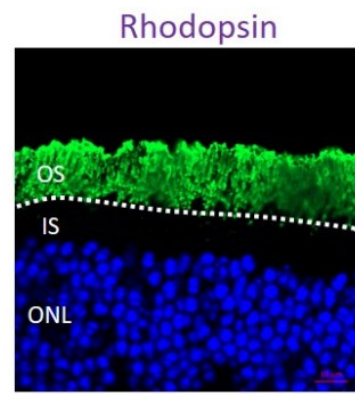

Red/Green opsin

Blue opsin

Transducin
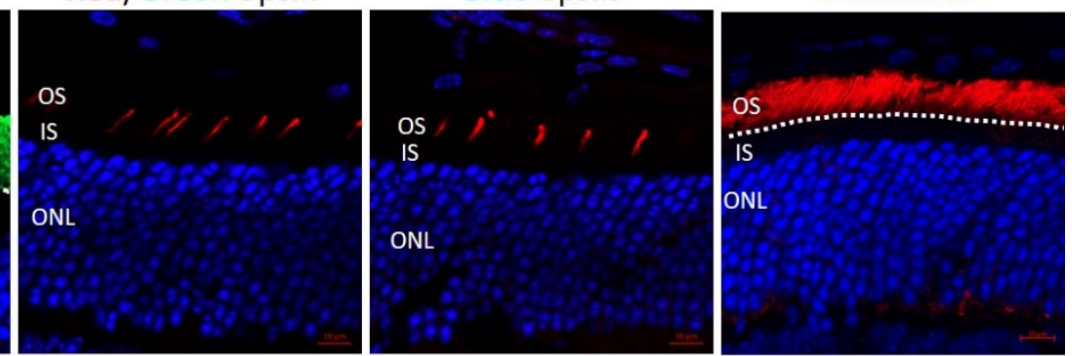

RPGR KO
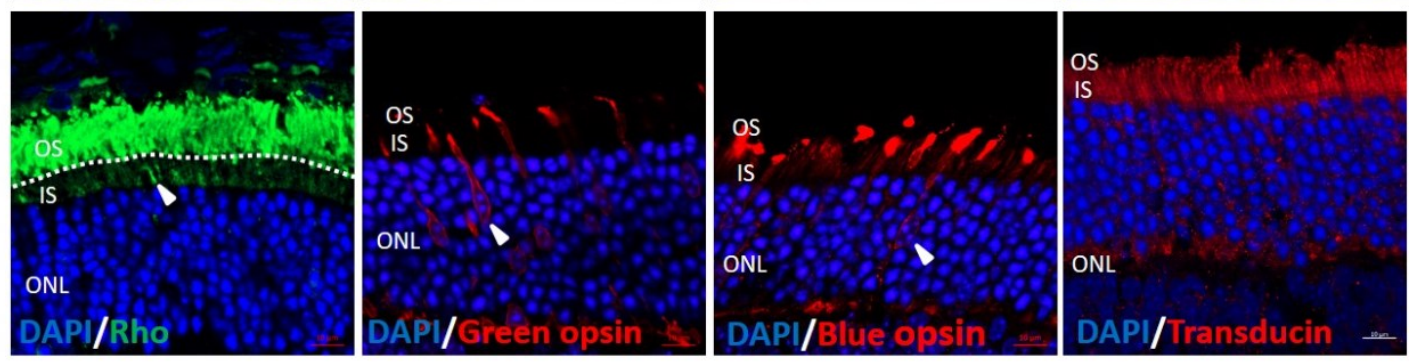

550

551

$552 \quad$ Figure 2

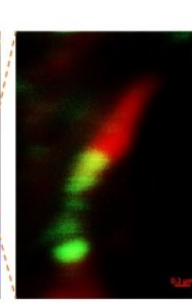

$235 \mathrm{kDa}$

$235 \mathrm{kDa} \quad$ WT 1M ко 1M WT 3M ко 3M WT 6M $\quad$ ко 6M WT 12M ко 12M

$235 \mathrm{kDa}$
$130 \mathrm{kDa}$
130

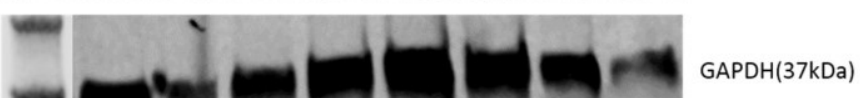

$36 \mathrm{kDa}$

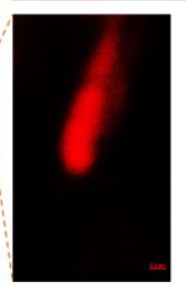

C

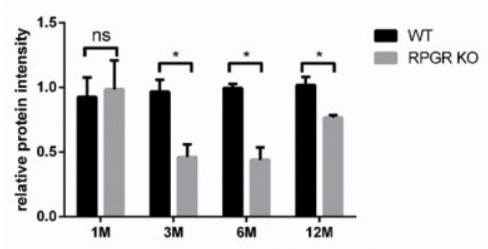

554

555

556

557 
$560 \quad$ Figure 3

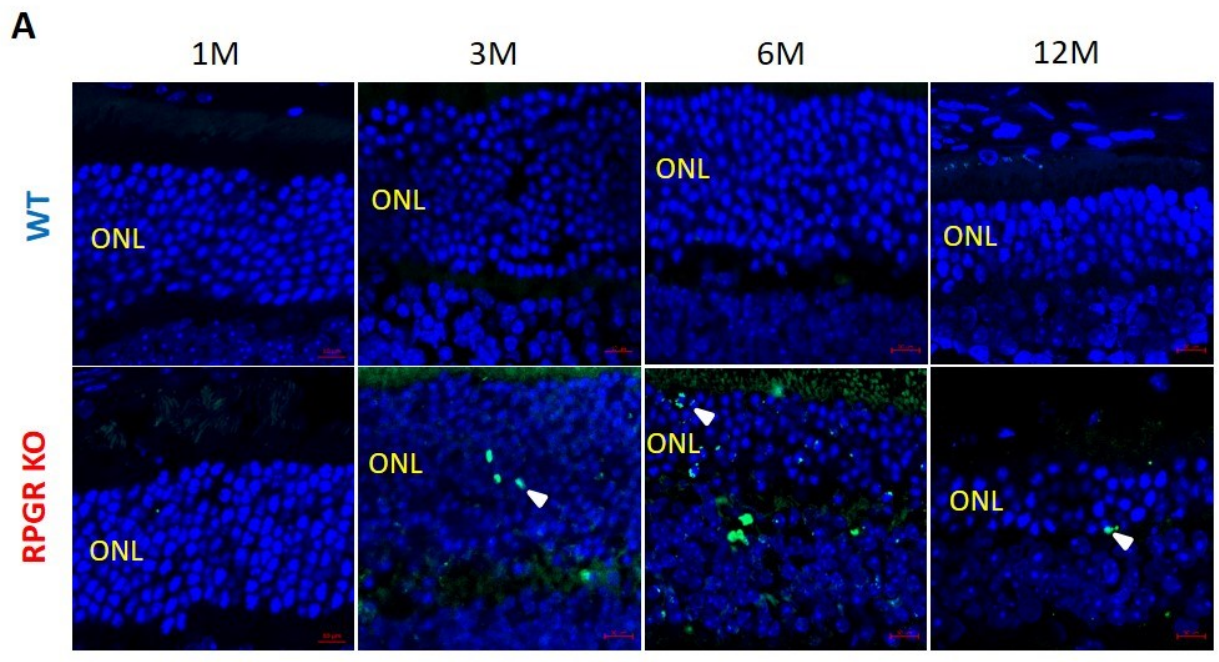

B

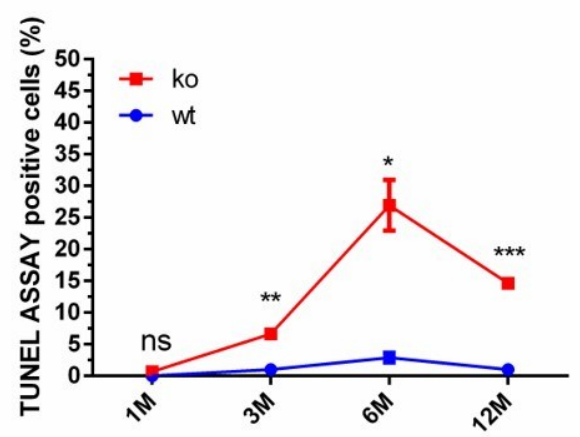

561

562

563

564

565

566

567

568

569

570

571

572 
$573 \quad$ Figure 4

A
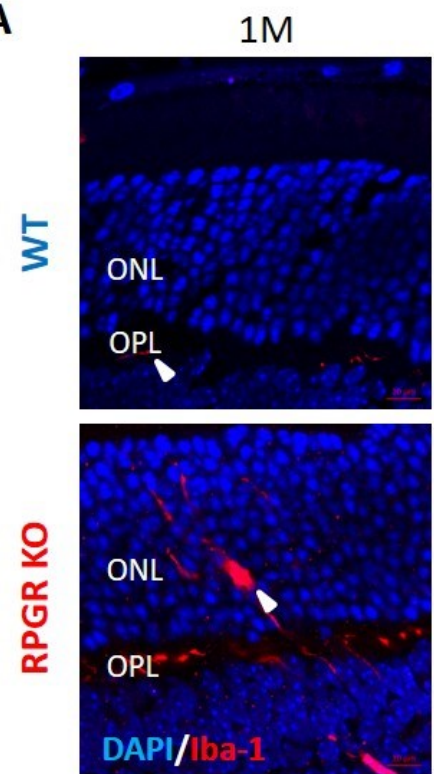

B

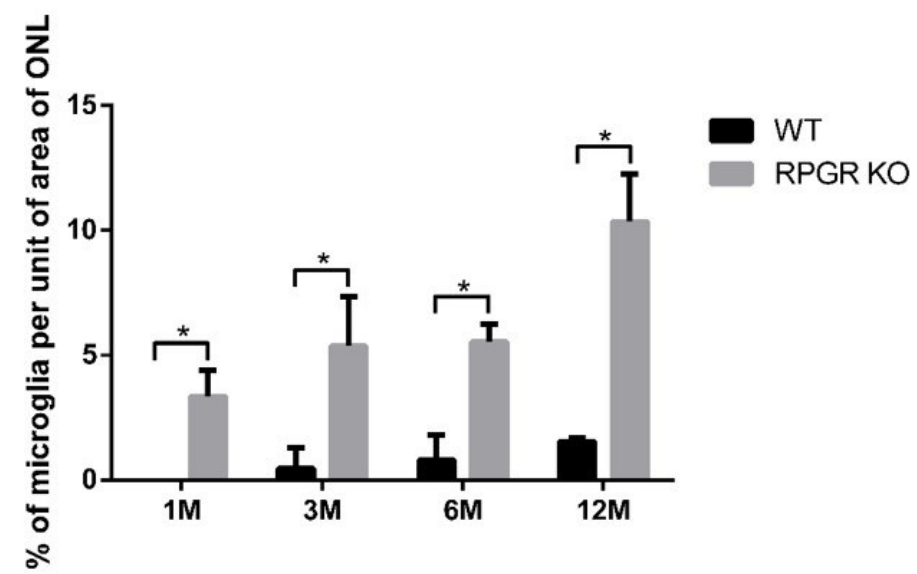

$6 \mathrm{M}$
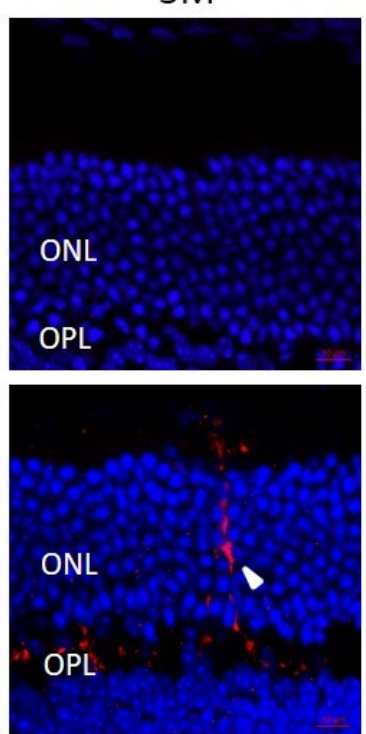
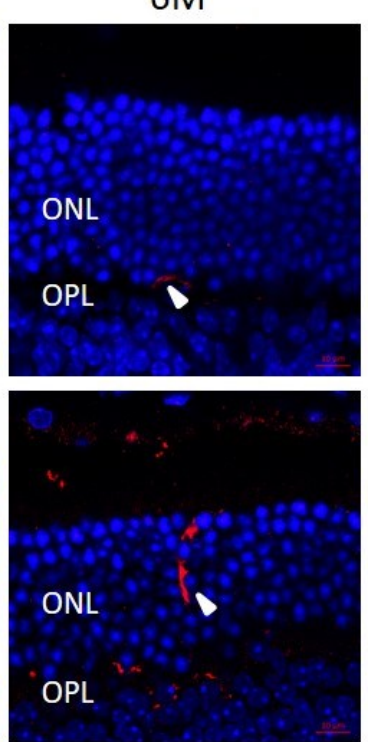

$12 \mathrm{M}$
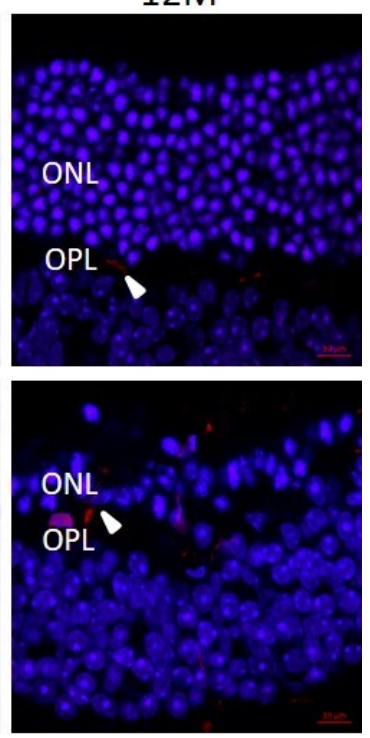
$583 \quad$ Figure 5

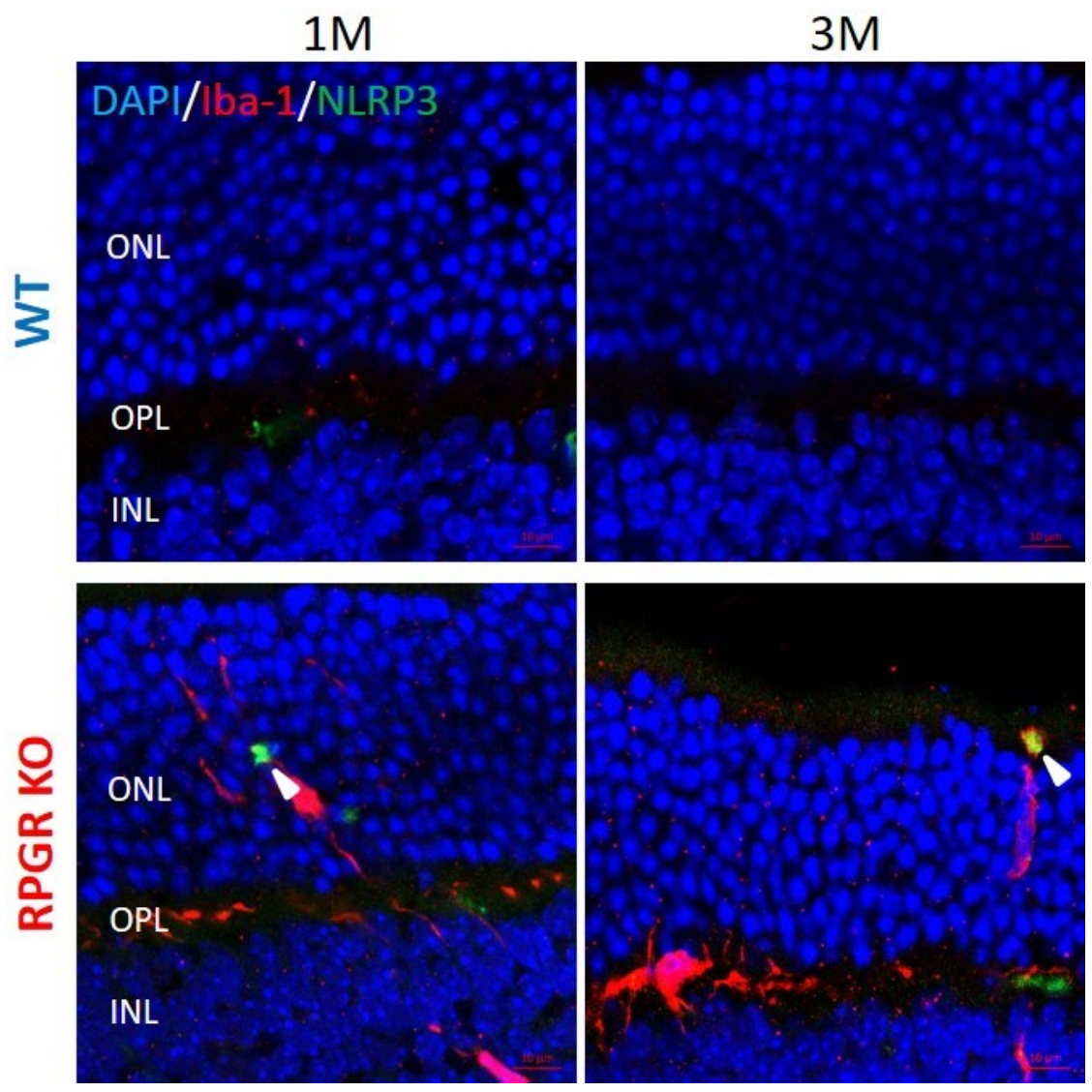

585

586

587

588

589

590

591

592

593

594

595

596 
Figure 6

A

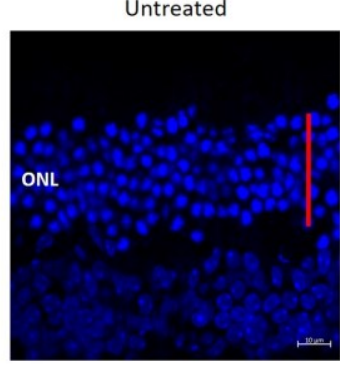

B

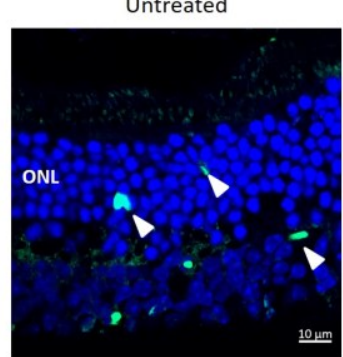

C

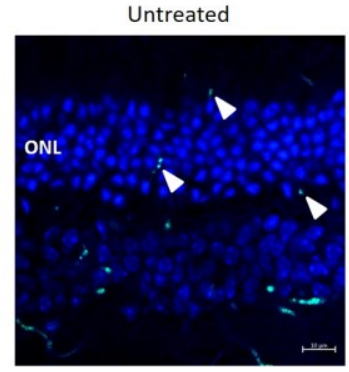

TUDCA treated

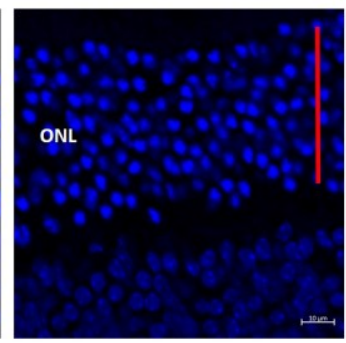

TUDCA treated

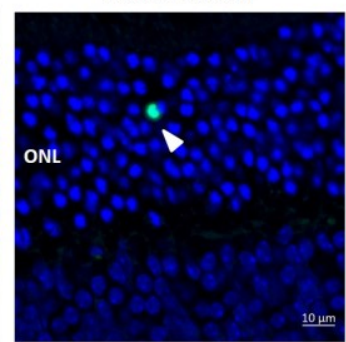

TUDCA treated

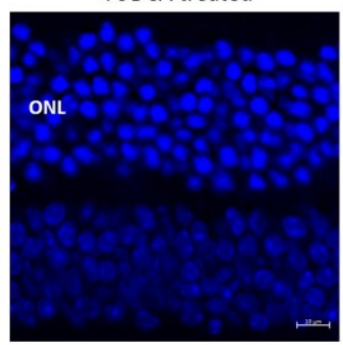

WT

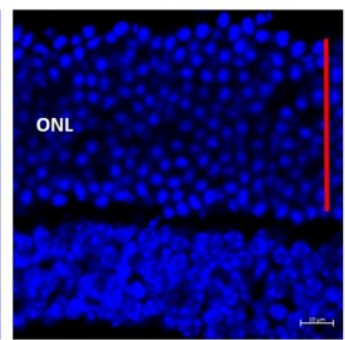

WT

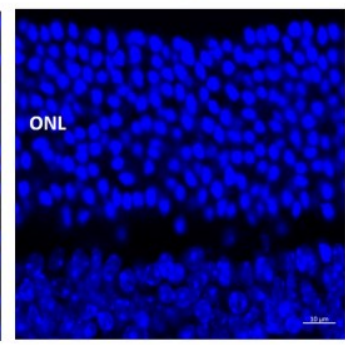

WT

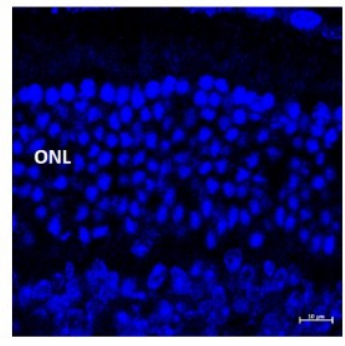

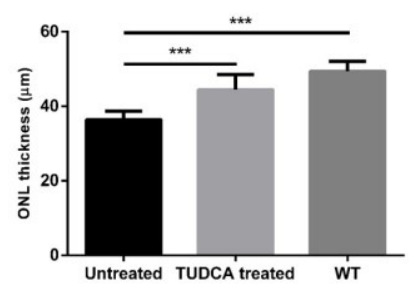
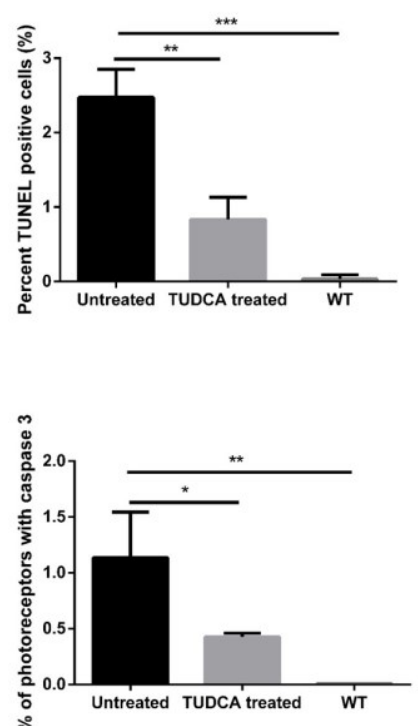

599

600

601

602

603

604

605

606

607

608

609

610 
$611 \quad$ Figure 7

A
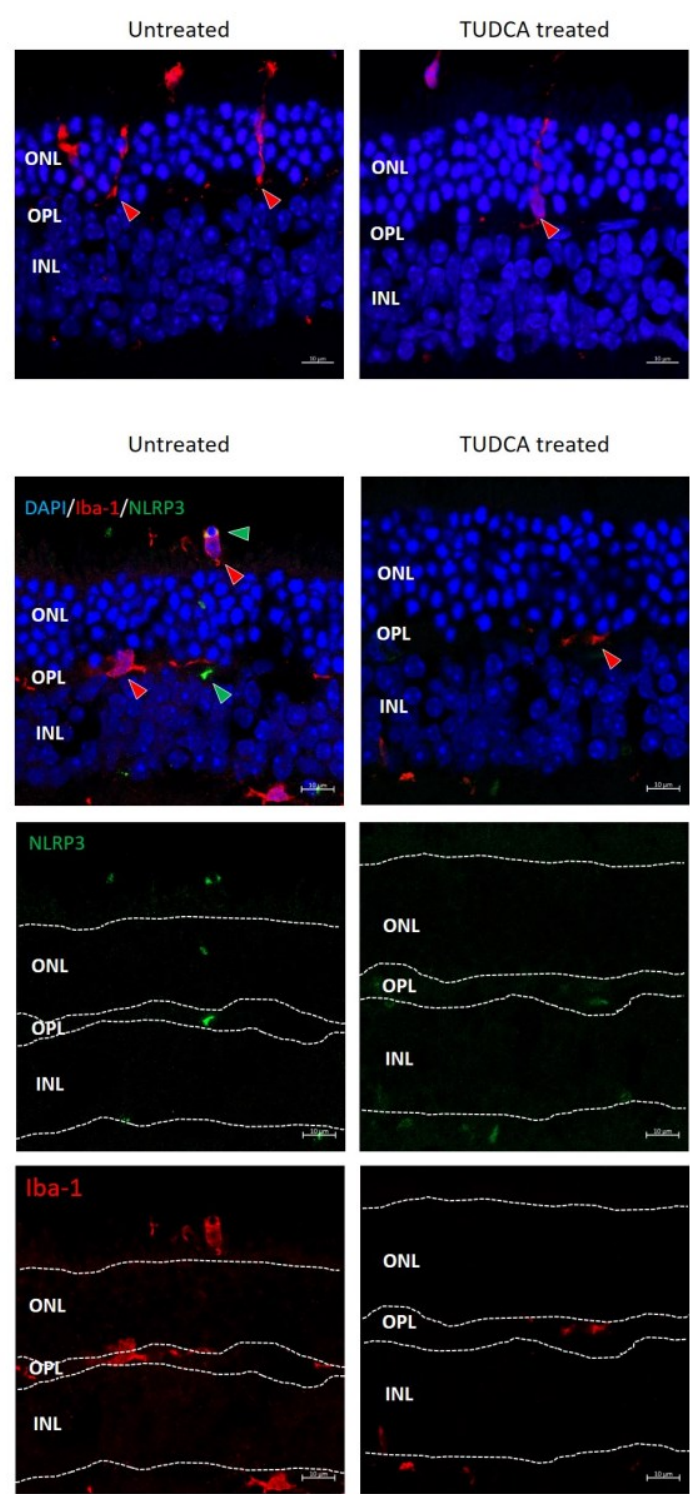

C

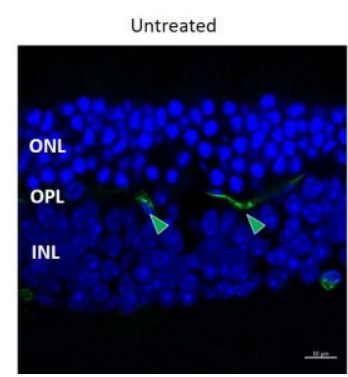

TUDCA treated
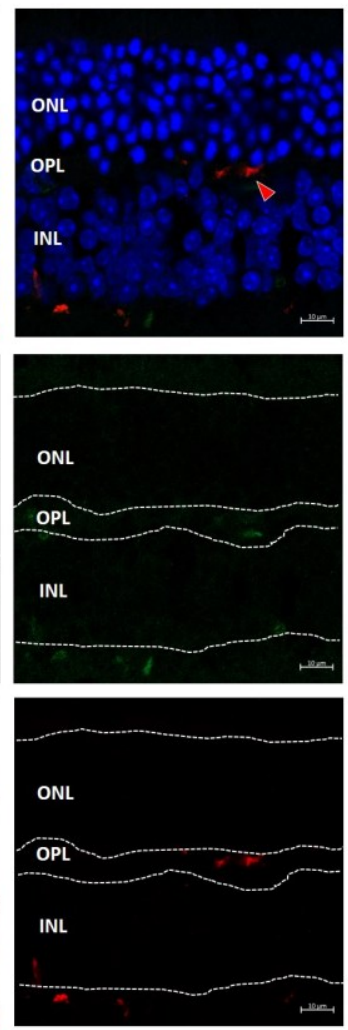

TUDCA treated

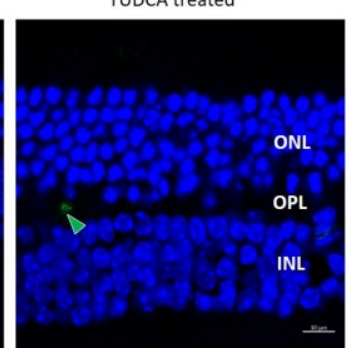

WT

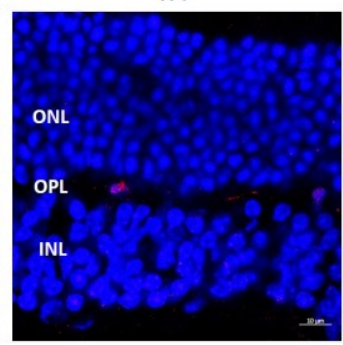

WT
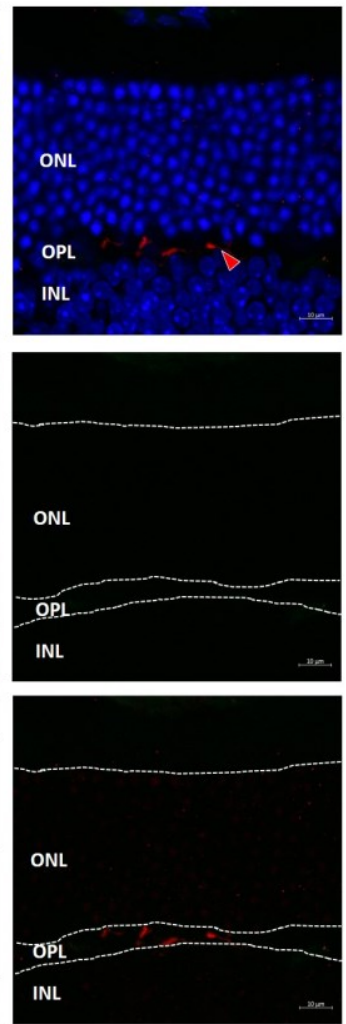

WT

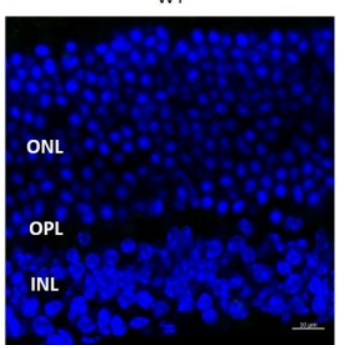

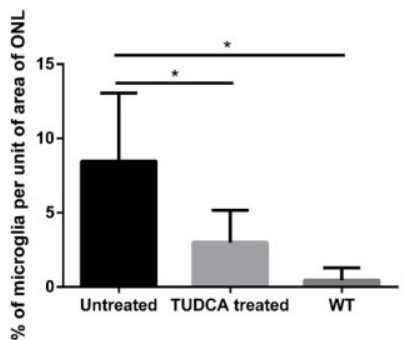

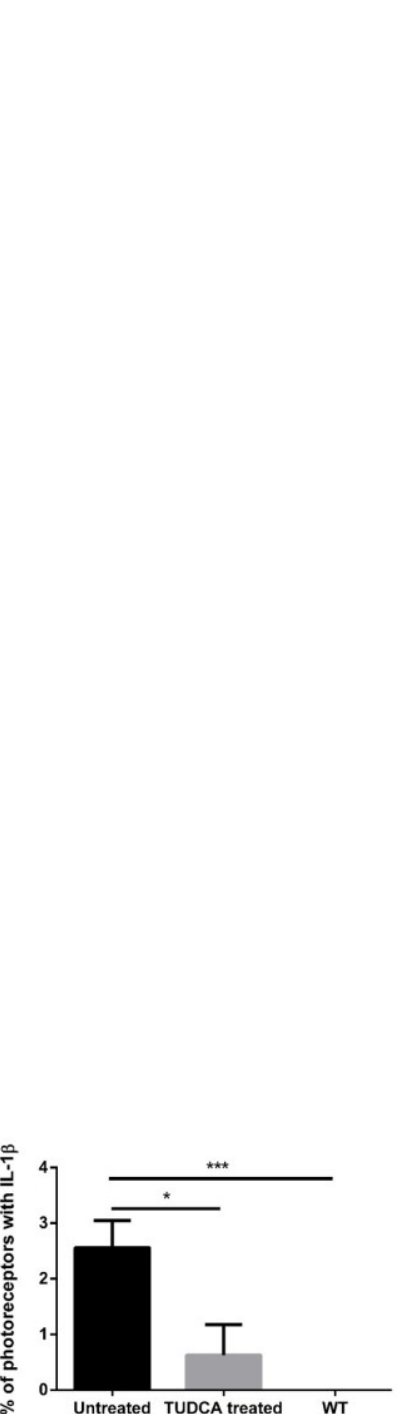

613

614

615 
618 Table S1. Primary antibodies were used for this study

\begin{tabular}{|c|c|c|}
\hline Primary Antibody & Dilution & Manufacturer \\
\hline Rhodopsin & $1: 200$ & Abcam, ab98887 \\
\hline M opsin & $1: 100$ & Abcam, ab5405 \\
\hline S opsin & $1: 100$ & Abcam, ab5407 \\
\hline$\alpha$-Transducin & $1: 100$ & Abcam, ab74059 \\
\hline GRK1 & $1: 100$ & Abcam, ab2775 \\
\hline NPHP4 & $1: 100$ & Antibody-online, ABIN1089278 \\
\hline Cleaved Caspase 3 & $1: 100$ & Cell Signaling \#9664 \\
\hline Iba-1 & $1: 100$ & Wako, 019-19741 \\
\hline NLRP3 & $1: 100$ & R\&D, MAB7578 \\
\hline IL-1 $\beta$ & $1: 200$ & Cell Signaling, \#12242 \\
\hline RPGR & $1: 100$ & Sigma, HPA001593 \\
\hline Acetylated $\alpha$ tubulin & $1: 200$ & Sigma, T6793 \\
\hline
\end{tabular}

619

620

621

622

623

624

625

626

627

628

629

630

631

632 
634 Figure S1 Full image of Western blot for Figure 2B. Description of the blot is provided in Figure 2B 635 legend.

636 Figure S2 Morphological change in Rpgr cko retinas. Histological examination following 637 hematoxylin and eosin staining showed decreased thickness of the outer nuclear layer (ONL) in Rpgr cko retina from 1 month (M) to 12M old. Green bars indicate ONL. Graphs show the thickness of ONL on both superior and inferior sides of retinas. Compared to wildtype (WT, blue line) retinas, the thickness of the ONL of Rpgr cko retinas (red line) was significantly decreased from 3months (M) old $(n=5)$. INL, inner nuclear layer; IS, inner segment; OPL, outer plexiform layer; OS, outer segment. Statistical comparisons were performed by a non-parametric $t$-test following by Wilcoxon matchedpairs signed rank test. ${ }^{*} \mathrm{p}<0.05$.

Figure S3 The rhodopsin signal (green) indicated the length of outer segment (OS) in retinas, represented by the double-headed red arrow. IS, inner segment; ONL, outer nuclear layer. (B) The length of outer segments (OS) of Rpgr cko (RPGR KO) retinas from 3 months (M) old was significantly reduced when compared to that of wildtype (WT) retinas. The length of OS at different ages was measured by ZEN and analysed by GraphPad Prism $(n=5)$. Statistical comparisons were performed by a non-parametric $t$-test following by Wilcoxon matched-pairs signed rank test. ${ }^{*} \mathrm{p}<0.05$. Figure S4 Caspase-dependent apoptosis was involved in photoreceptor degeneration in Rpgr cko retina. (A) Cryosections of retinas were stained for cleaved-caspase 3 (green), counterstaining with DAPI (blue). Caspase 3 was observed in the outer plexiform layer (OPL) of cko retinas from 3 months (M) old and also in the outer nuclear layer (ONL) at 6M and 12M old. In wildtype (WT) retina, no cleaved-caspase 3 was observed $(n=5)$.

(B) Quantification of cleaved-caspase 3 signal. Percentage of photoreceptors in which cleaved-caspase 3 signals were detected in total photoreceptors. Statistical comparisons were performed by a non-parametric $t$-test followed by Wilcoxon matchedpairs signed rank test. ${ }^{*} \mathrm{p}<0.05$.

Figure S5 Counting of photoreceptors in wildtype (WT), TUDCA-treated and untreated mouse 659 retinas. Cryosections of WT, TUDCA-treated and untreated mouse eyes were stained with DAPI 660 (blue). The results represent data obtained from two regions (one in the superior retina and one in the 
661 inferior retinas, $100 \mu \mathrm{m} \times 100 \mu \mathrm{m}$ under $400 \mathrm{x}$ magnification, $0.4 \mathrm{~mm}$ from optic nerve head, $\mathrm{ONH}$ ) of

662 two sections of each eye. Five eyes from five individual mice were used for the quantification. Data

663 were analyzed by GraphPad Prism with une-way ANOVA.

664 Figure S6 Expression of Caspase 3 and IL-1 $\beta$ (mRNA) was suppressed by TUDCA treatment.

665 TUDCA treatment significantly reduced the mRNA levels of Caspase 3 (A) and IL-1 $\beta$ (B) in retinas

666 of Rpgr cko mice. Statistical comparisons were performed using one-way ANOVA followed by

667 Bonferroni test. $* * \mathrm{p}<0.01, * * * \mathrm{p}<0.001$.

668

669

670

671

672

673

674

675

676

677

678

679

680

681

682

683

684

685

686

687

688 


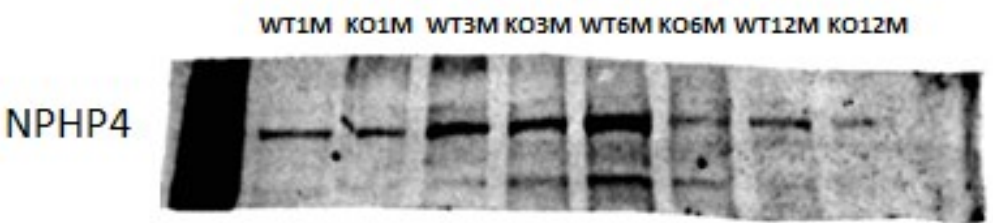

690

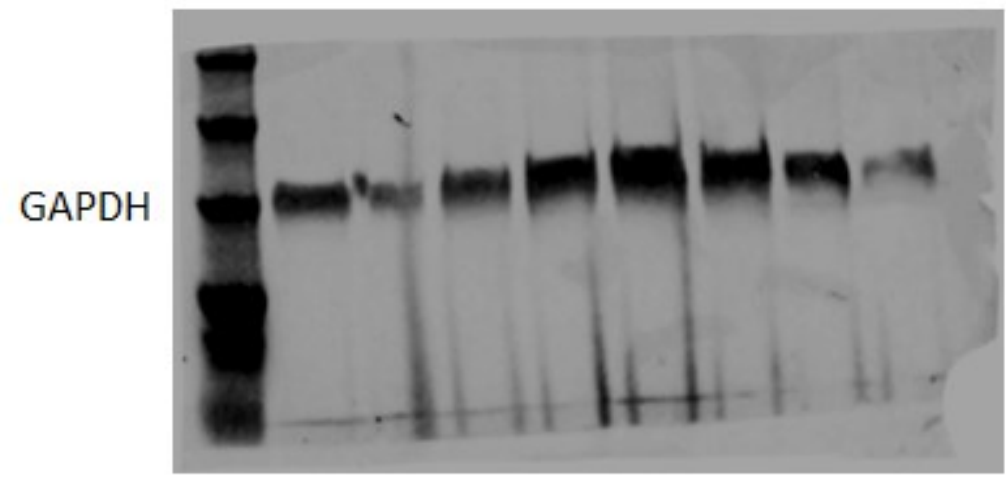

691

692

693

694

695

696

697

698

699

700

701

702

703

704

705

706

707 


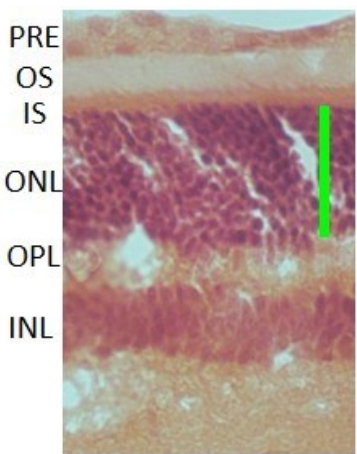

WT 1M

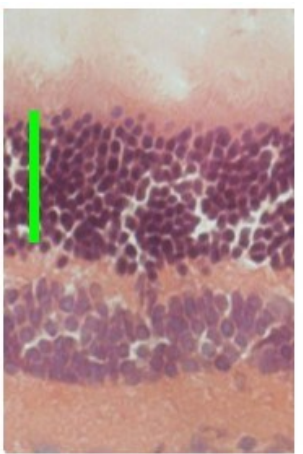

RPGR KO 1M

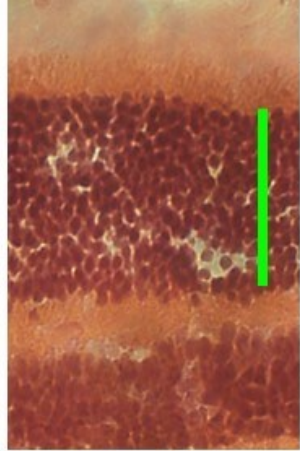

WT 3M

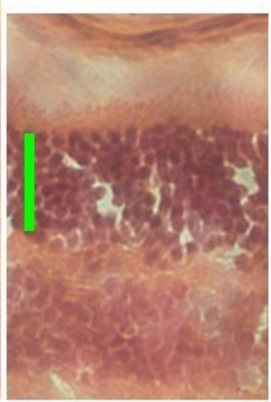

RPGR KO 3M
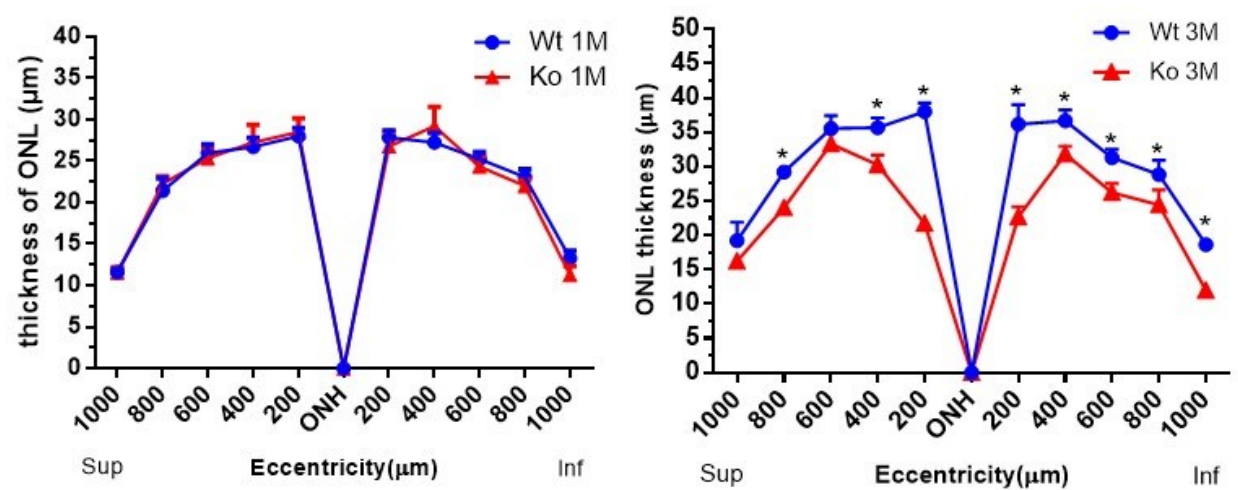

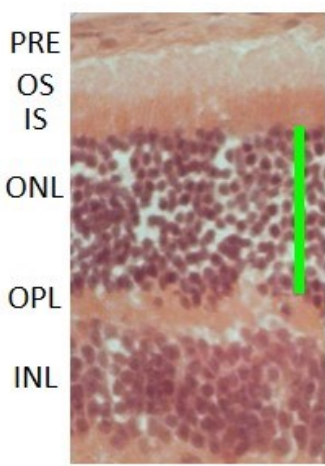

WT 6M

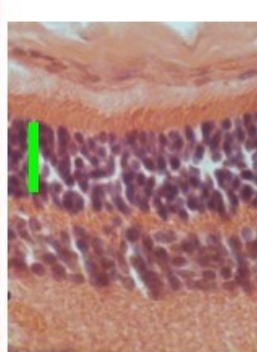

RPGR KO 6M

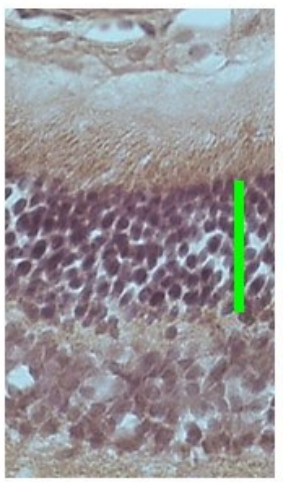

WT 12M RPGR KO 12M
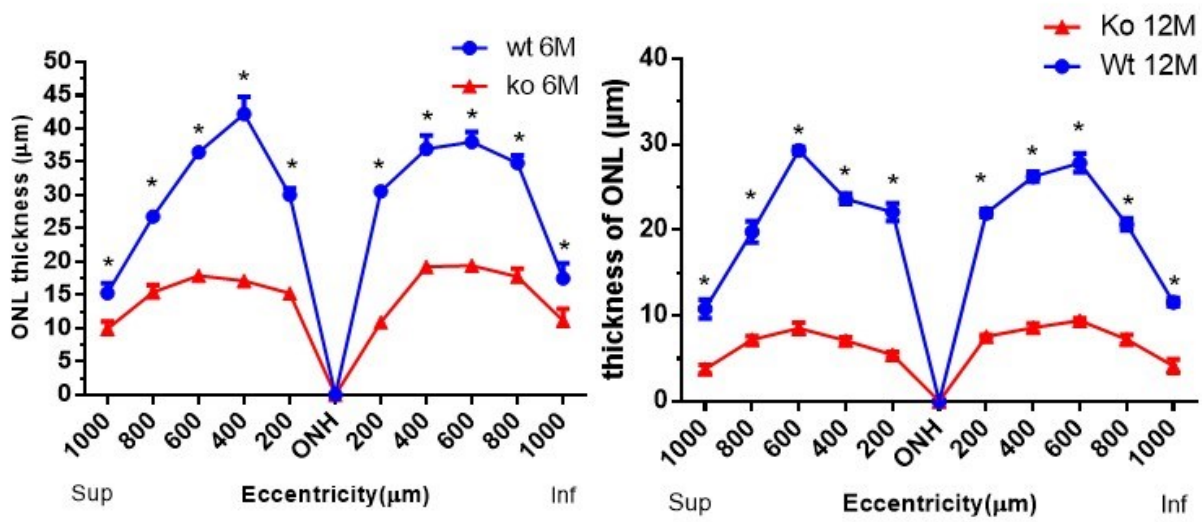
A
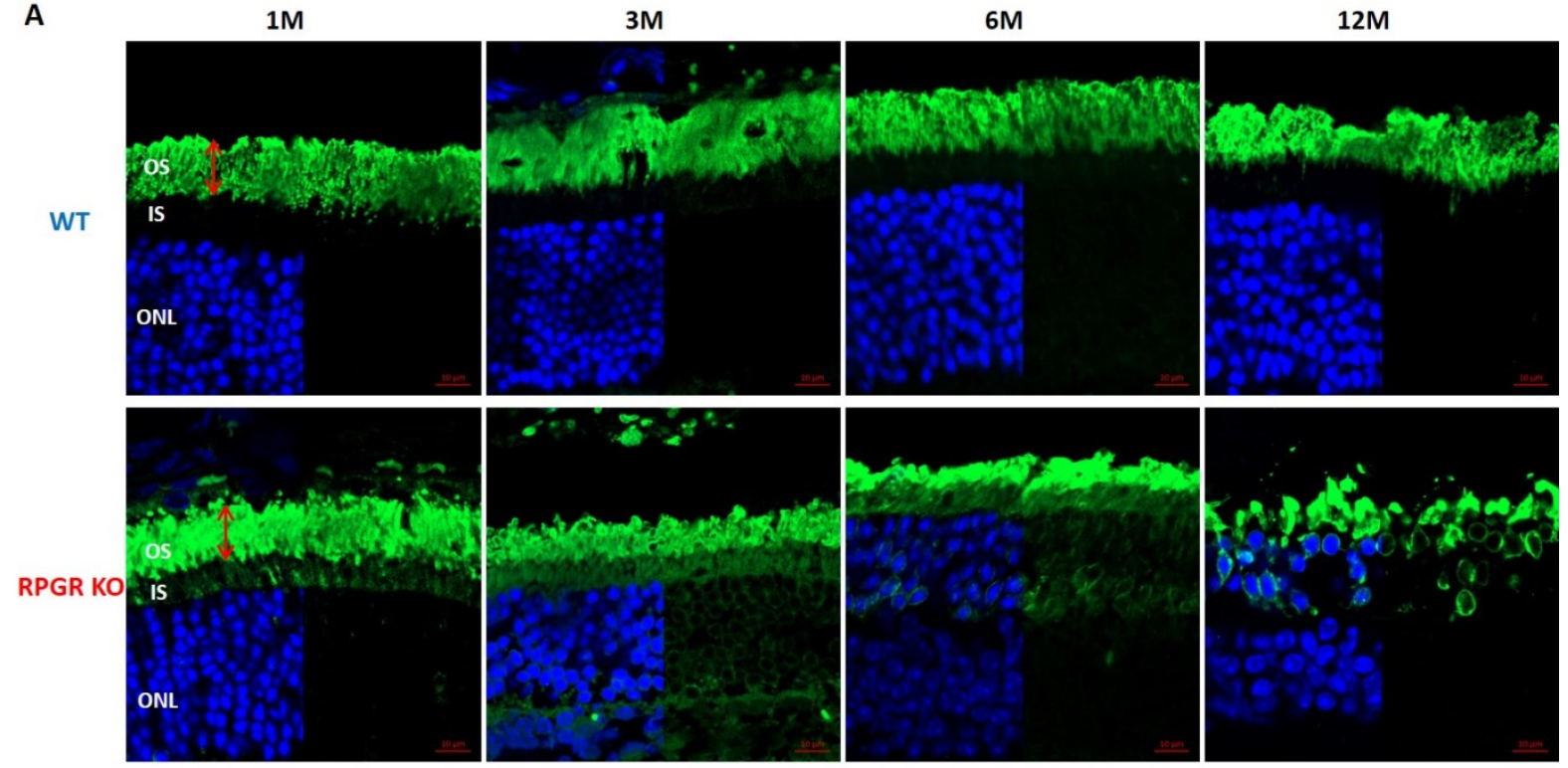

B

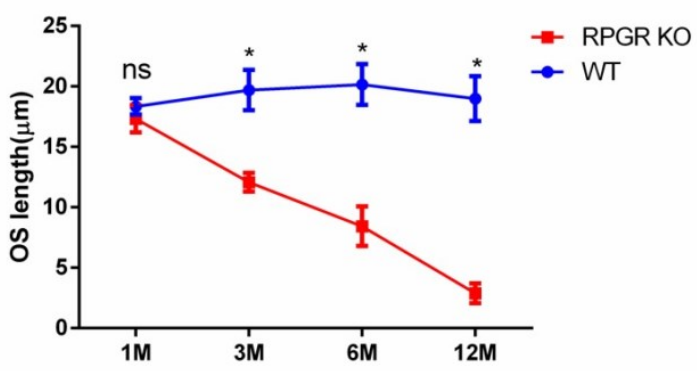

711 


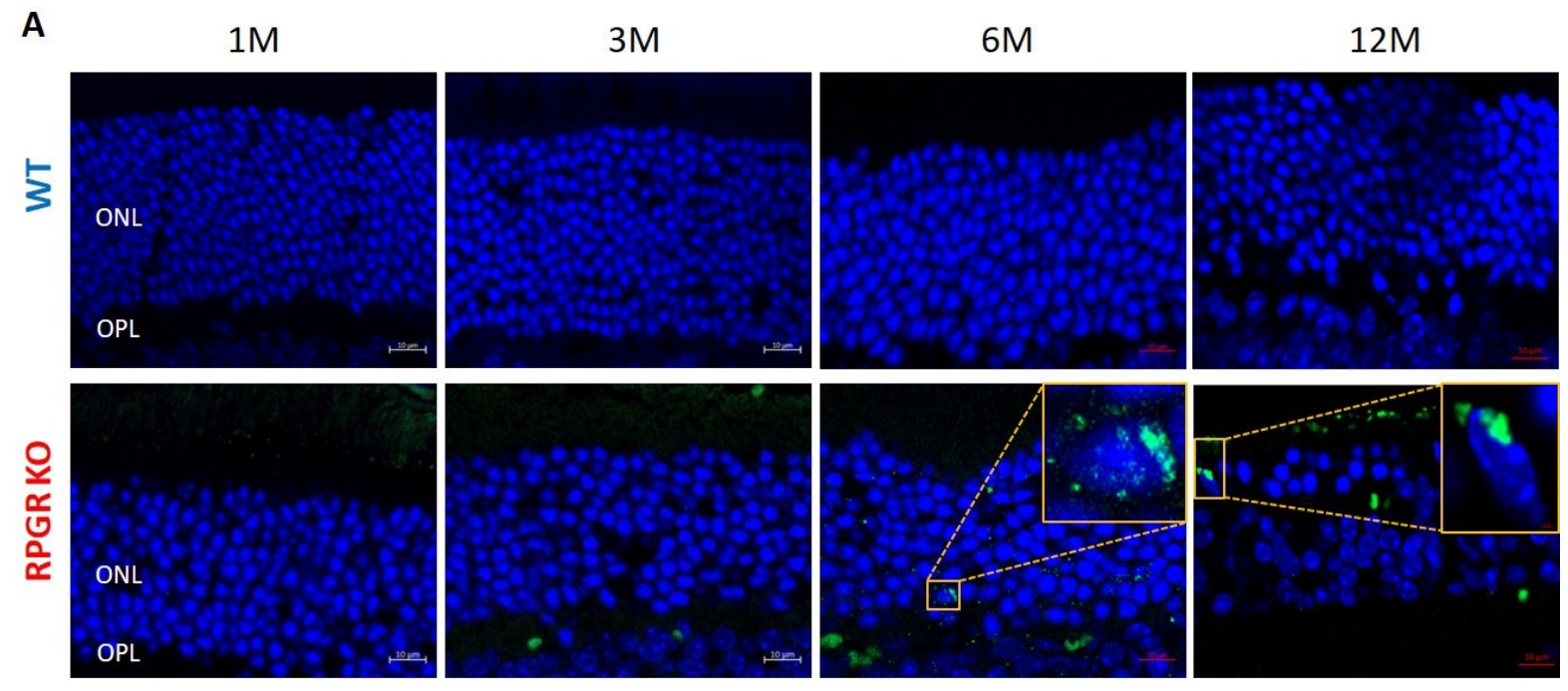

B

723

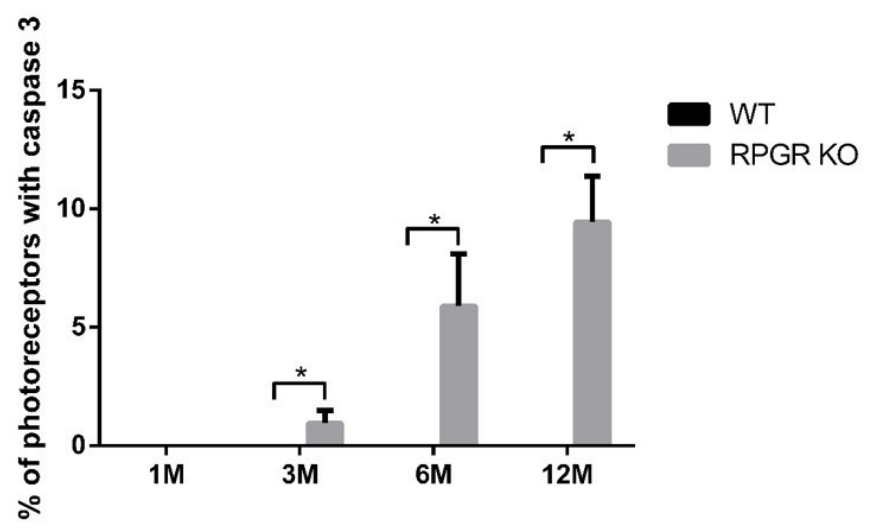

724

$725 \quad$ Figure S5

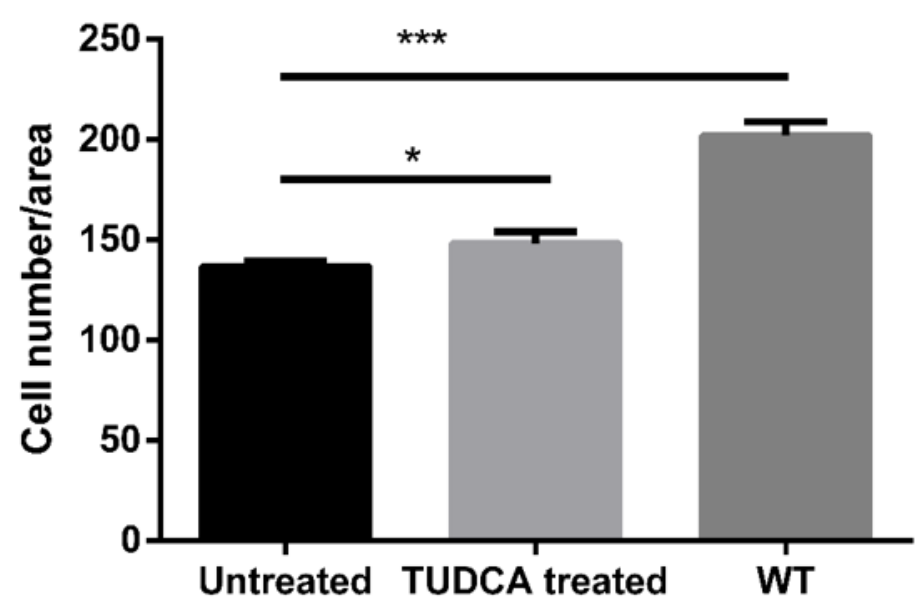


Figure S6

A

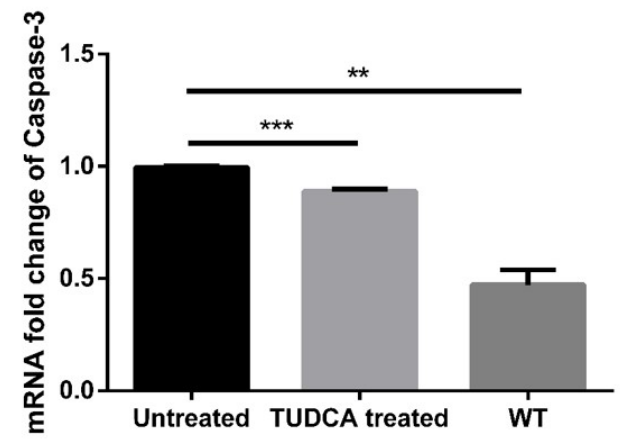

B

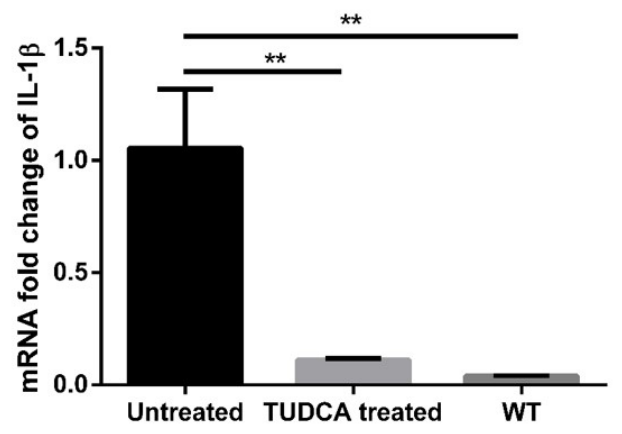

